\title{
Obesity and cancer immunotherapy toxicity
}

\author{
"The American Society of Clinical Oncology highlights that obesity \\ is quickly surpassing tobacco as a leading, preventable, cause of \\ cancer ... statistics underscore that the phenotype of the 'typical' \\ cancer patient may be changing."
}

Keywords: cancer $\bullet$ cytokines $\bullet$ immunotherapy $\bullet$ inflammation $\bullet$ obesity $\bullet$ toxicity

Within the past decade the usage of cancer immunotherapy has gained momentum, achieving successes within various advancedstaged malignancies and is beginning to be explored as a primary therapeutic rather than secondary to traditional cytoreductive therapies. Immunotherapeutic antitumor approaches have included systemic stimulatory therapies, blocking checkpoint inhibitory pathways, direct induction of T-cell stimulatory pathways and adoptive cell transfer strategies, among others [1]. However, the widespread application of immunotherapy has been limited by the induction of dose-limiting, and often times life-threatening, immune-related adverse reaction events (irAEs). These reactions are best exemplified by stimulatory systemic therapies, such as IL-2 and high-dose IFN, that can result in cytokine-release syndrome, multiorgan failure and require their usage to be limited to highly specialized centers capable of providing continuous care [2].

More recently, novel immunotherapies have similarly resulted in the induction of irAEs ranging from mild, requiring dose and scheduling adjustments, to life-threatening. Immune checkpoint blockade therapies such as anti-CTLA- 4 and anti-PD-1 induce a generalized expansion and activation of $\mathrm{T}$ cells that can adversely result in autoimmunity. Toxicities with checkpoint blockade thera- pies have included dermatitis, enterocolitis, endocrinopathies and life-threatening liver toxicities, and/or pneumonitis [3-5]. Likewise, a Phase II clinical trial using agonistic CD137 antibody to overcome T-cell inhibition through direct binding and activation of 4-1BB co-stimulatory receptor resulted in grade 4 hepatitis that halted the continuance of the trial [6]. Recently, great promise in antitumor efficacy has been demonstrated through autologous infusion of engineered $T$ cells that express chimeric antigen receptors (CARs). Low-dose administration of CAR-T cells expressing CD137 and targeting CD19 into three patients with chemotherapy-resistant chronic lymphocytic leukemia resulted in all three demonstrating strong antitumor responses with two patients having complete remission [7]. However, CAR-T-cell therapy is accompanied by cytokine release syndrome and/or macrophage activation syndrome that can culminate in the development of severe respiratory and cardiac dysfunction, requiring specialized care throughout treatment [7-9].

Of importance is the commonality that preclinical modeling has often failed to reveal toxicities that are observed in human clinical trials. The vast majority of preclinical studies make usage of young, lean, specific-pathogen free (SPF) inbred mouse models that are
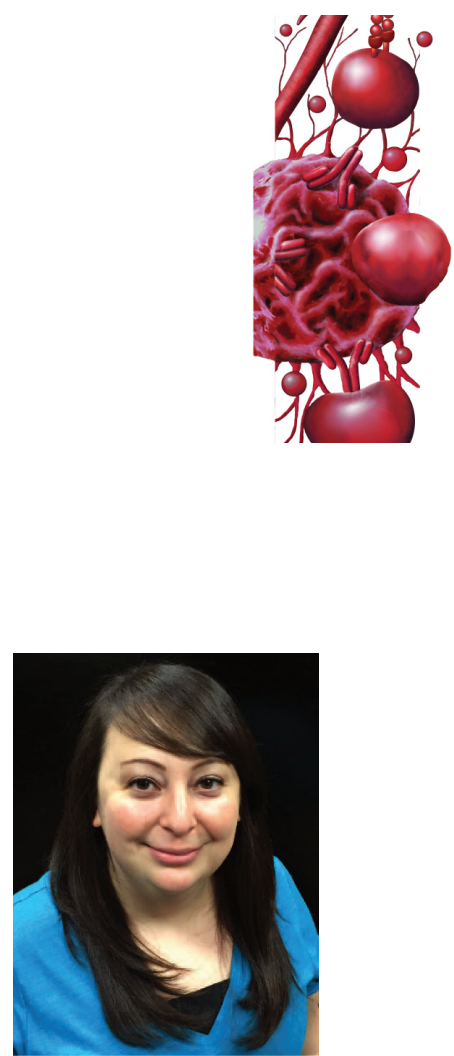

\section{Annie Mirsoian}

Department of Dermatology, University of California Davis, 2921 Stockton Blvd, IRC Suite 1630, Sacramento, CA 92817, USA

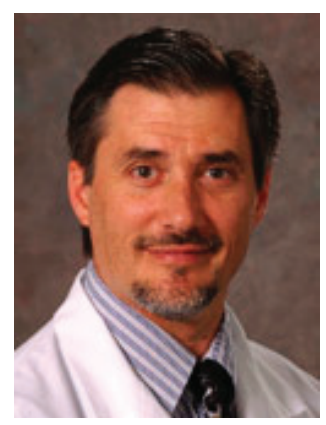

William J Murphy

Author for correspondence:

Department of Dermatology, University of California Davis, 2921 Stockton Blvd, IRC Suite 1630, Sacramento, CA 92817, USA

and

Department of Internal Medicine, University of California Davis, 2921 Stockton Blvd, IRC Suite 1610, Sacramento, CA 92817, USA Tel.: +1 9167039397 wmjmurphy@ucdavis.edu

Future $\because$ Medicine part of 
not reflective of the human cancer patient phenotype, which are usually much older. Another variable is body mass. Within the US the CDC estimates that greater than a third of all adults are currently obese (BMI $>30)$; a figure estimated to rise to be between $42-67 \%$ by 2030 [10]. Alarmingly, obesity is also becoming an epidemic of younger ages with a current estimated prevalence of $17 \%$ in children and adolescents [11]. In their 2014 position statement, the American Society of Clinical Oncology attributed obesity to be a major unrecognized risk factor for cancer. The American Society of Clinical Oncology highlights that obesity is quickly surpassing tobacco as a leading, preventable, cause of cancer [12]. These statistics underscore that the phenotype of the 'typical' cancer patient may be changing. Therefore, if the success of immunotherapy is contingent upon the activation and modulation of an immune response, then it is of the utmost importance to account for the baseline inflammatory profiles present in a cancer patient; as this may skew not only therapeutic responses but also affect toxicity risk. Together, this calls into question the relevancy of using young lean animal modeling in determining treatment toxicity, whereas changing the animal phenotype may provide more insightful data with increased translatability.

Although studies are lacking in investigating the ability of obesity to modulate immunotherapy responses, the pathogenesis of obesity is characterized by a heightened inflammatory environment that may prove critical to patient outcomes. Where adipocytes have traditionally been described as energy store houses, it has become evident that adipocytes play a larger bioactive role by serving as immune modulators, being able to secrete adipokines that serve a dual role as metabolic and immune regulators (i.e., leptin, IL-6) as well as by expressing a variety of immunologically relevant receptors such as Toll-like receptors, IL-6 receptor and TNF receptor [13]. Obesity is hallmarked by a self-sustaining inflammatory response termed 'meta-inflammation.' In contrast to classically defined acute inflammatory responses to injury or insult, meta-inflammation is nonresolving, localized within adipose depots and characteristically displays a chronic low-grade expression of proinflammatory mediators such as cytokines TNF- $\alpha$, IL-6 and IL-1 $\beta$; chemokines LTB-4, MCP-1 and MIP-1; and continual expression of proinflammatory transcription factors NF- $\mathrm{KB}$ and JNK [14]. The exact mechanistic initiation of meta-inflammation remains to be explicated, yet much literature has resolved that in both humans and mouse models adipose tissue macrophages in obesity converge with necrotic adipocytes, thereby releasing 'danger' signals as well as cytokines that perpetuate an acute inflammatory response [15]. In this manner, macrophages are able to gather around as crown-like structures and become further polarized into M1 macrophages [16,17]. Additionally, saturated fatty-acids released by adipocyte lipolysis can bind TLR4, thereby establishing a self-sustaining proinflammatory loop [16,17]. Although the involvement of adaptive immune cells in the pathogenesis of obesity remains unclear, it is well documented that obesity is accompanied by increased infiltrates of $\mathrm{CD}^{+}$and CD8 ${ }^{+}$T cells, NKT cells, B cells and DCs into white adipose tissues [18].

\section{"Although studies are lacking in investigating the ability of obesity to modulate immunotherapy responses, the pathogenesis of obesity is characterized by a heightened inflammatory environment that may prove critical to patient outcomes.}

Recently, our laboratory has demonstrated the importance of accounting for pre-existing inflammatory changes, as those induced with obesity, when administering immunotherapy regimens. We have previously demonstrated that systemic stimulatory immunotherapy administration, such as with agonistic monoclonal CD40 antibody in combination with IL-2 $(\alpha \mathrm{CD} 40 / \mathrm{IL}-2)$, induces antitumor responses and is well tolerated in young, lean mice (8-12 weeks of age). However, the administration of immunotherapy into aged (15 months and above) ad libitum fed mice resulted in the induction of a cytokine storm, as evidenced by high TNF- $\alpha$ and IL- 6 , that culminated in multiorgan pathological responses and rapid lethality [19]. Similar effects were observed with other stimulatory cytokine immunotherapies as well as with systemic LPS administration [19]. These results indicate that toxic responses are induced with strong immune stimulation, are not regimen specific and can be exacerbated by the pre-existing inflammatory environment of the patient. Upon characterization of the aged mice, we observed that ad libitum fed standard aged mice typically displayed markedly increased body weight that was largely due to increased visceral adiposity. Furthermore, we confirmed that obesity plays a critical role in the induction of immunotherapy toxicities as aged calorie-restricted mice treated with immunotherapy tolerated the therapy and demonstrated decreased cytokine levels that were either lower than or as-low-as young lean mice. Additionally, aged calorie-restricted mice demonstrated protection from multiorgan pathology [20]. Conversely, treatment of young obese mice (ob/ob and DIO) resulted in the induction of a cytokine storm similar to aged mice, as evidenced by heightened TNF- $\alpha$, multiorgan pathological responses, that were also confirmed by high 
ALT liver enzyme levels, and ultimately culminated in rapid lethality [20]. Given that obesity is hallmarked by chronically activated M1 macrophages and increased levels of proinflammatory cytokines, we investigated whether intervention by either macrophage depletion or administration of TNF-blockade could ameliorate the observed toxicities. Indeed, both macrophage depletion through systemic administration of liposomal clodronate or systemic TNF-blockade demonstrated complete protection of young obese mice and aged mice being treated with immunotherapy leading to $100 \%$ survival. Importantly, these interventions also allowed for immunotherapy regimens to induce successful antitumor effects in aged mice [19] indicating that blockade of inflammation does not necessarily affect antitumor efficacy.

“

"...the vast majority of studies within the context of obesity focus upon the innate immune response, and therefore very little to almost no data exists examining the consequences of obesity on molecular and cellular alterations to T-cell activation."

Our data demonstrates the significance of accounting for baseline levels of inflammation in determining toxicity risk as well as the critical importance of using preclinical models that mirror the human cancer scenario. It is important to note that strong immune stimulation resulted in a cytokine storm response in both our aged ad libitum and young obese mice, mimicking toxicities seen with systemic administration of IL-2 in cancer patients. Flow cytometric analysis of peritoneal lavages and visceral adipose tissues revealed that young obese mice exhibited greater M1/M2 ratios and when treated with immunotherapy contained higher percentages of TNF- $\alpha^{+}$macrophages in comparison to young

\section{References}

1 Mellman I, Coukos G, Dranoff G. Cancer immunotherapy comes of age. Nature 480(7378), 480-489 (2011).

2 Gangadhar TC, Vonderheide RH. Mitigating the toxic effects of anticancer immunotherapy. Nat. Rev. Clin. Oncol. 11(2), 91-99 (2014).

3 Topalian SL, Hodi FS, Brahmer JR et al. Safety, activity, and immune correlates of anti-PD-1 antibody in cancer. $N$. Engl. J. Med. 366(26), 2443-2454 (2012).

4 Weber JS, Kahler KC, Hauschild A. Management of immune-related adverse events and kinetics of response with ipilimumab. J. Clin. Oncol. 30(21), 2691-2697 (2012).

5 Robinson MR, Chan CC, Yang JC et al. Cytotoxic $\mathrm{T}$ lymphocyte-associated antigen 4 blockade in patients lean mice [20]. Our data imply that a phenotype with pre-existing inflammation may have deleterious consequences on systemic immunotherapy regimens, which may include CAR therapy as this form of therapy has reported development of macrophage activation syndrome in treated patients during infusions. It would be of benefit to retrospectively stratify treated patients by BMI to determine if toxicity severity correlates with pretreatment BMI.

However, the vast majority of studies within the context of obesity focus upon the innate immune response, and therefore very little to almost no data exists examining the consequences of obesity on molecular and cellular alterations to T-cell activation. Future studies need to address if this heightened inflammatory response also results when T-cell activation via checkpoint inhibitors are administered. Additionally, the concurrent presence of infection and/or infection history may exacerbate the inflammatory response as well, therefore accounting for not only baseline inflammation with obesity but also infection history is important in determining toxicity risk. The implications of a chronically activated inflammatory adaptive compartment within obesity may have consequences on the ability to mount antigen-specific responses leading to an overall dysregulation within this branch of immunity and, therefore, merits further evaluation.

\section{Financial \& competing interests disclosure}

The authors have no relevant affiliations or financial involvement with any organization or entity with a financial interest in or financial conflict with the subject matter or materials discussed in the manuscript. This includes employment, consultancies, honoraria, stock ownership or options, expert testimony, grants or patents received or pending, or royalties.

No writing assistance was utilized in the production of this manuscript. with metastatic melanoma: a new cause of uveitis. J. Immunother. 27(6), 478-479 (2004).

6 Ascierto PA, Simeone E, Sznol M, Fu YX, Melero I. Clinical experiences with anti-CD137 and anti-PD1 therapeutic antibodies. Semin. Oncol. 37(5), 508-516 (2010).

7 Porter DL, Levine BL, Kalos M, Bagg A, June CH. Chimeric antigen receptor-modified $\mathrm{T}$ cells in chronic lymphoid leukemia. N. Engl. J. Med. 365(8), 725-733 (2011).

8 Porter DL, Kalos M, Zheng Z, Levine B, June C. Chimeric antigen receptor therapy for B-cell malignancies. J. Cancer 2, 331-332 (2011).

9 Kochenderfer JN, Dudley ME, Feldman SA et al. B-cell depletion and remissions of malignancy along with cytokine-associated toxicity in a clinical trial of anti-CD19 chimeric-antigen-receptor-transduced T cells. Blood 119(12), 2709-2720 (2012). 
10 Prevention CFDCA (2012). Adult Obesity Facts. www.cdc.gov/obesity/data/adult.html

11 Prevention CFDCA (2013). Childhood Obesity Facts: Data and Statistics. www.cdc.gov/obesity/data/childhood.html

12 Ligibel JA, Alfano CM, Courneya KS et al. American Society of Clinical Oncology position statement on obesity and cancer. J. Clin. Oncol. 32(31), 3568-3574 (2014).

13 Ailhaud G. Adipose tissue as an endocrine organ. Int. J. Obes. Relat. Metab. Disord. 24(Suppl. 2), S1-S3 (2000).

14 Berg AH, Scherer PE. Adipose tissue, inflammation, and cardiovascular disease. Circ. Res. 96(9), 939-949 (2005).

15 Cinti S, Mitchell G, Barbatelli G et al. Adipocyte death defines macrophage localization and function in adipose tissue of obese mice and humans. J. Lipid Res. 46(11), 2347-2355 (2005)

16 Lee JY, Sohn KH, Rhee SH, Hwang D. Saturated fatty acids, but not unsaturated fatty acids, induce the expression of cyclooxygenase-2 mediated through Toll-like receptor 4 . J. Biol. Chem. 276(20), 16683-16689 (2001).

17 Suganami T, Tanimoto-Koyama K, Nishida J et al. Role of the Toll-like receptor 4/NF-kappaB pathway in saturated fatty acid-induced inflammatory changes in the interaction between adipocytes and macrophages. Arterioscler. Thromb. Vasc. Biol. 27(1), 84-91 (2007).

18 Kanneganti TD, Dixit VD. Immunological complications of obesity. Nat. Immunol. 13(8), 707-712 (2012).

19 Bouchlaka MN, Sckisel GD, Chen M et al. Aging predisposes to acute inflammatory induced pathology after tumor immunotherapy. J. Exp. Med. 210 (11), 2223-2237 (2013).

20 Mirsoian A, Bouchlaka MN, Sckisel, GD et al. Adiposity induces lethal cytokine storm after systemic administration of stimulatory immunotherapy regimens in aged mice. J. Exp. Med. 211(12), 2373-2383 (2014). 